\title{
Breakdown of Nonrelativistic QCD Factorization in Processes Involving Two Quarkonia and its Cure
}

\author{
Zhi-Guo He, Bernd A. Kniehl, and Xiang-Peng Wang \\ II. Institut für Theoretische Physik, Universität Hamburg, Luruper Chaussee 149, 22761 Hamburg, Germany
}

(Received 11 May 2018; revised manuscript received 18 August 2018; published 24 October 2018)

\begin{abstract}
We study inclusive processes involving two heavy quarkonia in nonrelativistic QCD (NRQCD) and demonstrate that, in the presence of two $P$-wave Fock states, NRQCD factorization breaks down, leaving uncanceled infrared singularities. As phenomenologically important examples, we consider the decay $\Upsilon \rightarrow \chi_{c J}+X$ via $b \bar{b}\left({ }^{3} P_{J_{b}}^{[8]}\right) \rightarrow c \bar{c}\left({ }^{3} P_{J}^{[1]}\right)+g g$ and the production process $e^{+} e^{-} \rightarrow J / \psi+\chi_{c J}+X$ via $e^{+} e^{-} \rightarrow c \bar{c}\left({ }^{3} P_{J_{1}}^{[8]}\right)+c \bar{c}\left({ }^{3} P_{J}^{[1]}\right)+g$. We infer that such singularities will appear for double quarkonium hadroproduction at next-to-leading order. As a solution to this problem, we introduce to NRQCD effective field theory new types of operators whose quantum corrections absorb these singularities.
\end{abstract}

DOI: 10.1103/PhysRevLett.121.172001

Because of their large mass scales and nonrelativistic nature, heavy-quarkonium states are ideal probes to study quantum chromodynamics (QCD), which is the fundamental theory to describe the strong interactions between quarks and gluons, from both perturbative and nonperturbative aspects. The traditional color-singlet model (CSM), which restricts the heavy-quark pair, $Q \bar{Q}$, to form a color singlet and to share the spectroscopic quantum numbers ${ }^{2 S+1} L_{J}$ of spin $S$, orbital angular momentum $L$, and total angular momentum $J$ with the physical quarkonium state, is plagued by infrared (IR) singularities when applied to the production or decay of $P$-wave quarkonia [1] or quarkonia with $L>1$ [2]. Phenomenologically, a cutoff, e.g., on the binding energy of the $Q \bar{Q}$ bound state or the momenta of emitted gluons, has to be introduced to regularize such singularities, which makes the theoretical predictions model dependent and causes the separation of short- and long-distance physics to break down. This problem has been successfully solved by the factorization formalism [3] built on the rigorous effective field theory of nonrelativistic QCD (NRQCD) [4]. In the NRQCD factorization formalism [3], the production and decay rates of heavy quarkonia are separated into short-distance coefficients (SDCs), which can be obtained as expansions in the strong-coupling constant $\alpha_{s}$ through the NRQCD to full-QCD matching of perturbative calculations, and supposedly universal longdistance matrix elements (LDMEs). The sizes of the latter are subject to scaling rules in the velocity $v_{Q}$ of $Q$ and $\bar{Q}$ in

Published by the American Physical Society under the terms of the Creative Commons Attribution 4.0 International license. Further distribution of this work must maintain attribution to the author(s) and the published article's title, journal citation, and DOI. Funded by SCOAP ${ }^{3}$. the $Q \bar{Q}$ rest frame [5]. This allows us to calculate heavyquarkonium production and decay rates systematically as double expansions in $\alpha_{s}$ and $v_{Q}^{2}$. The NRQCD factorization formalism has successfully cured the IR problem of the CSM, as explicitly shown in the literature for inclusive decay and production of $P$ - [6] and $D$-wave states [7]. Note that in exclusive processes, such as $B$-meson exclusive decay to $\chi_{c J}$ mesons [8] and exclusive double quarkonium production in $e^{+} e^{-}$annihilation [9], there are still uncanceled IR divergences at one loop, which disappear in the limits $m_{c} / m_{b} \rightarrow 0$ and $m_{c} / \sqrt{s} \rightarrow 0$, respectively [9].

Besides inclusive production and decay processes involving a single quarkonium state, processes in which two heavy quarkonia participate, such as bottomonium decay to charmonium or double heavy-quarkonium production, are also of great phenomenological interest. Measurements of $\Upsilon$ decay to charmonium, like the $J / \psi$ meson, can be traced to the first experiment carried out by the CLEO Collaboration about 30 years ago [10], and they were then updated by the ARGUS [11] and CLEO [12] Collaborations with much larger data samples. Very recently, more precise results for the branching ratios of $\Upsilon \rightarrow J / \psi\left(\psi^{\prime}\right)+X$ [13] and $\Upsilon \rightarrow \chi_{c 1}+X$ [14] were obtained by the Belle Collaboration. On the other hand, $J / \psi$ pair and $J / \psi+\Upsilon$ associated production have been a very hot topic at hadron colliders in recent years. In fact, double $J / \psi(\Upsilon)$ and $J / \psi+\Upsilon$ prompt production were extensively measured by the D0 Collaboration [15] at the FNAL Tevatron and by the LHCb [16], CMS [17], and ATLAS [18] Collaborations at the CERN LHC. Interestingly, there are substantial discrepancies between CMS data and NRQCD predictions at leading order (LO) in $\alpha_{s}$, which are expected to be reduced by the yet unknown next-to-leading-order (NLO) corrections [19]. Moreover, 
double heavy-quarkonium production serves as a useful laboratory to investigate the double parton scattering mechanism [20] at hadron colliders. On the theoretical side, both bottomonium decay to charmonium and double heavy-quarkonium production have been studied in the NRQCD factorization framework. In the former case, only the color-singlet (CS) ${ }^{3} S_{1}^{[1]}[21]$ and color-octet (CO) ${ }^{3} S_{1}^{[8]}$ [22] $b \bar{b}$ Fock states were considered. In the latter case, the CS contributions are known to NLO in $\alpha_{s}$ [23] and $v_{Q}^{2}$ [24], while the CO contributions are only known to LO [19] and partially to NLO in $v_{Q}^{2}$ [24]. In all these calculations, IR singularities appearing in intermediate steps were always properly removed by NRQCD factorization, and one might have expected that this is a general rule valid for all $Q \bar{Q}$ Fock states and to all orders in $\alpha_{s}$ and $v_{Q}^{2}$.

In this Letter, we change this familiar picture by presenting two counterexamples, suggesting that the well-established formalism needs a generalization for the cases at hand. In fact, the violation of NRQCD factorization appears as one includes more $Q \bar{Q}$ Fock states or goes beyond LO in $\alpha_{s}$. As we demonstrate later on, the leftover IR singularities may, fortunately, be completely absorbed into the QCD corrections to a class of operators introduced here for the first time.

Let us first consider the inclusive production of charmonium $H=J / \psi, \chi_{c J}, \psi^{\prime}$ by $\Upsilon$ decay. By NRQCD factorization, the decay width can be expressed as

$$
\begin{aligned}
\Gamma(\Upsilon \rightarrow H+X) \\
\quad=\sum_{m, n} \hat{\Gamma}(b \bar{b}(m) \rightarrow c \bar{c}(n)+X)\langle\Upsilon|\mathcal{O}(m)| \Upsilon\rangle\left\langle\mathcal{O}^{H}(n)\right\rangle,
\end{aligned}
$$

where $m$ and $n$ are $b \bar{b}$ and $c \bar{c}$ Fock states, respectively, $\hat{\Gamma}(b \bar{b}(m) \rightarrow c \bar{c}(n)+X)$ are the SDCs, and $\langle\Upsilon|\mathcal{O}(m)| \Upsilon\rangle$ and $\left\langle\mathcal{O}^{H}(n)\right\rangle$ are the LDMEs. According to the velocity scaling rules [5], for $\Upsilon\left(J / \psi, \psi^{\prime}\right)$ the four Fock states ${ }^{3} S_{1}^{[1]}$, ${ }^{1} S_{0}^{[8]},{ }^{3} S_{1}^{[8]}$, and ${ }^{3} P_{J}^{[8]}$ contribute through relative order $v_{b}^{4}$ $\left(v_{c}^{4}\right)$, while for $\chi_{c J}$ the dominant contributions come from ${ }^{3} P_{J}^{[1]}$ and ${ }^{3} S_{1}^{[8]}$ at order $v_{c}^{2}$. We have checked explicitly that, in the single $P$-wave case, where either $b \bar{b}$ or $c \bar{c}$ is in a $P$-wave state, the IR singularities arising in the full QCD calculation can be completely absorbed into the corresponding $S$-wave LDMEs rendering $\hat{\Gamma}(b \bar{b}(m) \rightarrow c \bar{c}(n)+$ $X)$ IR finite. However, when both $b \bar{b}$ and $c \bar{c}$ are in $P$-wave states, there are extra IR singularities that cannot be absorbed into the $S$-wave LDMEs. At LO, there are two such subprocesses, namely $b \bar{b}\left({ }^{3} P_{J_{b}}^{[8]}\right) \rightarrow c \bar{c}\left({ }^{3} P_{J_{c}}^{[8]}\right)+g g$ for $\Upsilon \rightarrow J / \psi+X$ and $b \bar{b}\left({ }^{3} P_{J_{b}}^{[8]}\right) \rightarrow c \bar{c}\left({ }^{3} P_{J_{c}}^{[1]}\right)+g g$ for $\Upsilon \rightarrow$ $\chi_{c J}+X$. In the following, we focus our attention on the second one. Similar conclusions can be drawn for the first one and can eventually be extended to the general case of bottomonium decay to charmonium plus anything.

There are eight Feynman diagrams for $b \bar{b}\left({ }^{3} P_{J_{b}}^{[8]}\right) \rightarrow$ $c \bar{c}\left({ }^{3} P_{J_{c}}^{[1]}\right)+g g$, and typical ones are depicted in Fig. 1 . For each choice of $J_{b}$ and $J_{c}$, the corresponding partonic decay rate may be calculated by using appropriate spin and color projectors. Unsurprisingly, they all contain IRdivergent terms. Because of space limitation, we present here only the latter. Furthermore, we sum over $J_{b}$ using heavy-quark spin symmetry, leaving $\left\langle\Upsilon\left|\mathcal{O}\left({ }^{3} P_{0}^{[8]}\right)\right| \Upsilon\right\rangle$ as the overall $\Upsilon$ LMDE. We write the result as

$$
\hat{\Gamma}^{\mathrm{div}}\left(J_{c}\right)=\hat{\Gamma}_{1}^{\mathrm{div}}+9 \hat{\Gamma}_{2}^{\mathrm{div}}\left(J_{c}\right)+\hat{\Gamma}_{3}^{\mathrm{div}}\left(J_{c}\right),
$$

where $\hat{\Gamma}_{1}^{\mathrm{div}}\left(\hat{\Gamma}_{2}^{\mathrm{div}}\left(J_{c}\right)\right)$ arises from the square of the amplitude $\mathcal{M}_{1}\left(\mathcal{M}_{2}\right)$ of the diagrams in which the soft gluon is emitted by the $c$ or $\bar{c}(b$ or $\bar{b})$ quarks, and $\hat{\Gamma}_{3}^{\mathrm{div}}\left(J_{c}\right)$ arises from the interference of $\mathcal{M}_{1}$ and $\mathcal{M}_{2}$. We have:

$$
\begin{aligned}
\hat{\Gamma}_{1}^{\text {div }}= & \frac{-8 \alpha_{s}}{27 \pi m_{c}^{2}} \frac{1}{\epsilon_{\mathrm{IR}}} \times \frac{5 \pi^{2} \alpha_{s}^{3}\left(3 r^{4}+2 r^{2}+7\right)}{72 m_{b}^{7} r^{3}\left(1-r^{2}\right)}, \\
\hat{\Gamma}_{2}^{\text {div }}\left(J_{c}\right)= & \frac{-5 \alpha_{s}}{9 \pi m_{b}^{2}} \frac{1}{\epsilon_{\mathrm{IR}}} \times\left\{\begin{array}{l}
\frac{\pi^{2} \alpha_{s}^{3}\left(1-3 r^{2}\right)^{2}}{81 m_{b}^{2} r^{3}\left(1-r^{2}\right)}, \quad J_{c}=0, \\
\frac{2 \pi^{2} \alpha_{s}^{3}\left(r^{2}+1\right)}{8 m_{b}^{7} r^{3}\left(1-r^{2}\right)}, \quad J_{c}=1, \\
\frac{2 \pi_{s}^{2} \alpha_{s}^{3}\left(6 r^{4}+3 r^{2}+1\right)}{405 m_{b}^{7} r^{3}\left(1-r^{2}\right)}, \quad J_{c}=2,
\end{array}\right. \\
\hat{\Gamma}_{3}^{\text {div }}(0)= & -\frac{10 \pi \alpha_{s}^{4}}{81 m_{b}^{9} r^{3}\left(1-r^{2}\right)^{4} \epsilon_{\mathrm{IR}}} \\
& \times\left(3 r^{4}-10 r^{2}+3\right)\left(r^{4}-4 r^{2} \ln r-1\right), \\
\hat{\Gamma}_{3}^{\text {div }}(1)= & \frac{10 \pi \alpha_{s}^{4}}{81 m_{b}^{9} r^{3}\left(1-r^{2}\right)^{4} \epsilon_{\mathrm{IR}}}\left[-r^{6}+9 r^{4}\right. \\
& \left.-7 r^{2}+4 r^{2}\left(r^{4}-3 r^{2}-2\right) \ln r-1\right], \\
\hat{\Gamma}_{3}^{\text {div }}(2)= & \frac{2 \pi \alpha_{s}^{4}}{81 m_{b}^{9} r^{3}\left(1-r^{2}\right)^{4} \epsilon_{\mathrm{IR}}}\left[6 r^{8}+23 r^{6}\right. \\
& \left.-27 r^{4}+r^{2}-4 r^{4}\left(9 r^{2}+11\right) \ln r-3\right],
\end{aligned}
$$

where $\epsilon_{\mathrm{IR}}=D / 2-2$ is the infrared regulator of dimensional regularization and $r=m_{c} / m_{b}$. In Eq. (3), we write

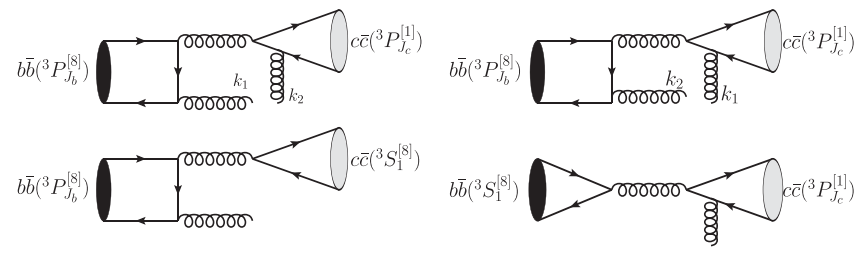

FIG. 1. Typical Feynman diagrams for $b \bar{b}\left({ }^{3} P_{J_{b}}^{[8]}\right) \rightarrow c \bar{c}\left({ }^{3} P_{J_{c}}^{[1]}\right)+$ $g g$ (upper panel), $b \bar{b}\left({ }^{3} P_{J_{b}}^{[8]}\right) \rightarrow c \bar{c}\left({ }^{3} S_{1}^{[8]}\right)+g$ (lower left panel), and $b \bar{b}\left({ }^{3} S_{1}^{[8]}\right) \rightarrow c \bar{c}\left({ }^{3} P_{J_{c}}^{[1]}\right)+g$ (lower right panel). 
$\hat{\Gamma}_{1}^{\text {div }}$ and $\hat{\Gamma}_{2}^{\text {div }}\left(J_{c}\right)$ as products of the SDCs of $b \bar{b}\left({ }^{3} P_{J_{b}}^{[8]}\right) \rightarrow$ $c \bar{c}\left({ }^{3} S_{1}^{[8]}\right)+g$ and $b \bar{b}\left({ }^{3} S_{1}^{[8]}\right) \rightarrow c \bar{c}\left({ }^{3} P_{J_{c}}^{[1]}\right)+g$, whose representative Feynman diagrams are shown in Fig. 1 as well, and the IR-singular terms arising from the NLO QCD corrections to the LDMEs $\left.\left\langle\mathcal{O}^{\chi_{c J}(}{ }^{3} S_{1}^{[8]}\right)\right\rangle$ and $\left\langle\Upsilon\left|\mathcal{O}\left({ }^{3} S_{1}^{[8]}\right)\right| \Upsilon\right\rangle$, respectively, so as to indicate that they will be canceled after taking into account the contributions of $b \bar{b}\left({ }^{3} P_{J_{b}}^{[8]}\right) \rightarrow$ $c \bar{c}\left({ }^{3} S_{1}^{[8]}\right)+g$ and $b \bar{b}\left({ }^{3} S_{1}^{[8]}\right) \rightarrow c \bar{c}\left({ }^{3} P_{J_{c}}^{[1]}\right)+g$. However, in the case of $\hat{\Gamma}_{3}^{\text {div }}\left(J_{c}\right)$, the NRQCD factorization formalism as we know it simply lacks an operator that could compensate the soft-gluon effects. This renders $\hat{\Gamma}^{\mathrm{div}}\left(J_{c}\right)$ IR singular altogether. In other words, we are faced by an IR problem of NRQCD factorization, which has gone unnoticed so far. Since $\hat{\Gamma}_{3}^{\mathrm{div}}\left(J_{c}\right)$ are due to the interference of diagrams with soft gluons emitted by $P$-wave $b \bar{b}$ and $c \bar{c}$ Fock states, which appear in NRQCD treatments of any inclusive decay of bottomonium to charmonium at some order of $v_{b}^{2}$ and $v_{c}^{2}$, we conclude that the NRQCD factorization formalism, in its familiar and generally accepted form, will break down for any such process. In some cases, this may happen even at relative order $v_{b, c}^{2}$, i.e., at LO, e.g., for the decay $\chi_{b J_{b}} \rightarrow \chi_{c J_{c}}+X$ via the channel $b \bar{b}\left({ }^{3} P_{J_{b}}^{[1]}\right) \rightarrow c \bar{c}\left({ }^{3} P_{J_{c}}^{[1]}\right)+g g$. We note in passing that, unlike for the exclusive processes mentioned above $[8,9], \hat{\Gamma}_{3}^{\mathrm{div}}\left(J_{c}\right)$ does not vanish in the limit $r \rightarrow 0$.

We now turn to our second example, the inclusive production of two heavy quarkonia, e.g., $2 J / \psi$, $J / \psi+\Upsilon$, etc. In the NRQCD treatment of $J / \psi$ pair or $J / \psi+\Upsilon$ associated hadroproduction, soft-gluon emission starts from NLO in $\alpha_{s}$, e.g., $g g \rightarrow c \bar{c}\left({ }^{3} P_{J_{1}}^{[8]}\right)+c \bar{c}\left({ }^{3} P_{J_{2}}^{[8]}\right)+g$. A complete NLO NRQCD calculation lies beyond the scope of this Letter. Instead, we choose a relatively simple process for illustration, namely $e^{+} e^{-} \rightarrow J / \psi+\chi_{c J}+X$

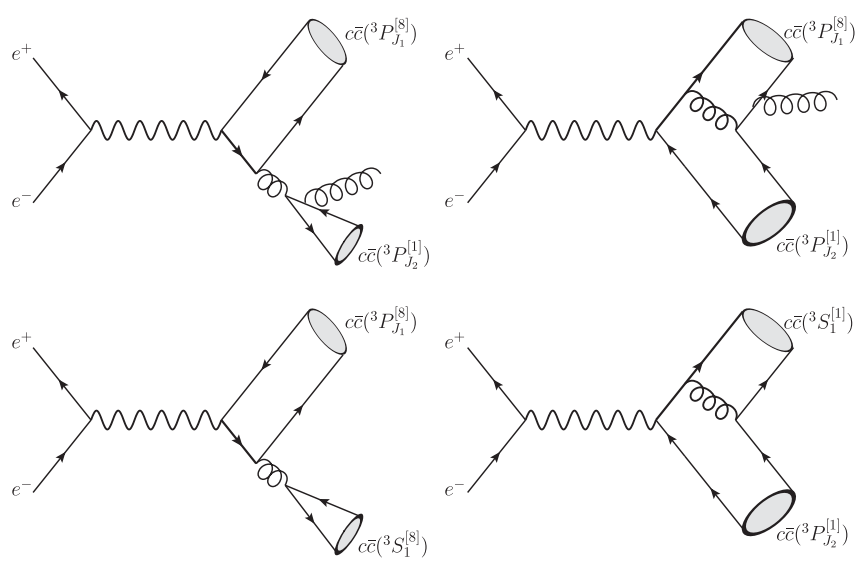

FIG. 2. Typical Feynman diagrams for $e^{+} e^{-} \rightarrow c \bar{c}\left({ }^{3} P_{J_{1}}^{[8]}\right)+$ $c \bar{c}\left({ }^{3} P_{J_{2}}^{[1]}\right)+g$ (upper panel), $e^{+} e^{-} \rightarrow c \bar{c}\left({ }^{3} P_{J_{1}}^{[8]}\right)+c \bar{c}\left({ }^{3} S_{1}^{[8]}\right)$ (lower left panel), and $e^{+} e^{-} \rightarrow c \bar{c}\left({ }^{3} S_{1}^{[1]}\right)+c \bar{c}\left({ }^{3} P_{J_{2}}^{[1]}\right)$ (lower right panel).

proceeding via the LO channel $e^{+} e^{-} \rightarrow c \bar{c}\left({ }^{3} P_{J_{1}}^{[8]}\right)+$ $c \bar{c}\left({ }^{3} P_{J_{2}}^{[1]}\right)+g$. The IR problem featured here should also appear in double $J / \psi$ production via $e^{+} e^{-}$ annihilation [25].

There are 28 contributing Feynman diagrams, typical ones of which are displayed in Fig. 2. Again, there are three sources of IR singularities in the full NRQCD calculation: (1) the square of the part of the amplitude where the gluon is attached to the $c \bar{c}\left({ }^{3} P_{J_{1}}^{[8]}\right)$ pair, (2) the same for ${ }^{3} P_{J_{2}}^{[1]}$, and (3) the interference of these two amplitude parts. After summing over $J_{1}$, we have for the IR-singular pieces

$$
\hat{\sigma}^{\mathrm{div}}\left(J_{2}\right)=\sigma_{1}^{\mathrm{div}}+9 \sigma_{2}^{\mathrm{div}}\left(J_{2}\right)+\sigma_{3}^{\mathrm{div}}\left(J_{2}\right),
$$

with

$$
\begin{aligned}
\hat{\sigma}_{1}^{\text {div }} & =-\frac{8 \alpha_{s}}{27 \pi m_{c}^{2}} \frac{1}{\epsilon_{\mathrm{IR}}} \times \frac{2^{10} \pi^{3} \alpha^{2} \alpha_{s}^{2} S}{3^{6} s^{5} r^{6}}\left(864 r^{10}-144 r^{8}-1568 r^{6}+1224 r^{4}-130 r^{2}+27\right), \\
\hat{\sigma}_{2}^{\text {div }}\left(J_{2}\right) & =-\frac{4 \alpha_{s}}{3 \pi m_{c}^{2}} \frac{1}{\epsilon_{\mathrm{IR}}} \times \frac{2^{18} \pi^{3} \alpha^{2} \alpha_{s}^{2} S}{3^{9} s^{5} r^{4}} \begin{cases}\left(144 r^{8}+152 r^{6}-428 r^{4}+182 r^{2}+1\right), & J_{2}=0, \\
8\left(18 r^{6}+13 r^{4}-12 r^{2}+2\right), & J_{2}=1, \\
\frac{2}{5}\left(360 r^{8}+308 r^{6}-188 r^{4}+20 r^{2}+1\right), & J_{2}=2,\end{cases} \\
\hat{\sigma}_{3}^{\text {div }}(0) & =\frac{2^{18} \pi^{2} \alpha^{2} \alpha_{s}^{3}}{3^{8} s^{6} r^{4} \epsilon_{\mathrm{IR}}}\left[\left(144 r^{8}+184 r^{6}-504 r^{4}+170 r^{2}+33\right) S+8\left(72 r^{10}+56 r^{8}-284 r^{6}+149 r^{4}+r^{2}\right) T\right], \\
\hat{\sigma}_{3}^{\text {div }}(1) & =\frac{2^{18} \pi^{2} \alpha^{2} \alpha_{s}^{3}}{3^{8} s^{6} r^{2} \epsilon_{\mathrm{IR}}}\left[\left(144 r^{6}+28 r^{4}-176 r^{2}+43\right) S+\left(576 r^{10}-176 r^{8}-792 r^{6}+424 r^{4}-48 r^{2}\right) T\right], \\
\hat{\sigma}_{3}^{\text {div }}(2) & =\frac{2^{18} \pi^{2} \alpha^{2} \alpha_{s}^{3}}{5 \cdot 3^{8} s^{6} r^{4} \epsilon_{\mathrm{IR}}}\left[\left(720 r^{8}+452 r^{6}-696 r^{4}+7 r^{2}-15\right) S+\left(2880 r^{10}+368 r^{8}-3560 r^{6}+1856 r^{4}-56 r^{2}\right) T\right],
\end{aligned}
$$


where $r=2 m_{c} / \sqrt{s}, \sqrt{s}$ is the center-of-mass energy, $S=\left(1-4 r^{2}\right)^{1 / 2}$, and $T=\tanh ^{-1} S$. It is straightforward to check that $\hat{\sigma}_{1}^{\text {div }}$ and $\hat{\sigma}_{2}^{\text {div }}\left(J_{2}\right)$ will be canceled after including the $\mathcal{O}\left(\alpha_{s}\right)$ corrections to the $S$-wave LDMEs $\left\langle\mathcal{O}^{\chi_{c J}}\left({ }^{3} S_{1}^{[8]}\right)\right\rangle$ and $\left\langle\mathcal{O}^{J / \psi}\left({ }^{3} S_{1}^{[1]}\right)\right\rangle$ in the SDCs of $e^{+} e^{-} \rightarrow$ $c \bar{c}\left({ }^{3} P_{J_{1}}^{[8]}\right)+c \bar{c}\left({ }^{3} S_{1}^{[8]}\right)$ and $e^{+} e^{-} \rightarrow c \bar{c}\left({ }^{3} S_{1}^{[1]}\right)+c \bar{c}\left({ }^{3} P_{J_{2}}^{[1]}\right)$, respectively. Typical Feynman diagrams for the latter two processes are also show in Fig. 2. Unfortunately, the standard NRQCD factorization formalism does not provide an intrinsic mechanism to cancel $\sigma_{3}^{\mathrm{div}}\left(J_{2}\right)$, and thus it fails to yield an IR-finite result for the cross section of $e^{+} e^{-} \rightarrow$ $J / \psi+\chi_{c J}+X$ at relative order $v_{c}^{4}$. Because $\sigma_{3}^{\mathrm{div}}\left(J_{2}\right)$ originates from the interference of Feynman diagrams where the gluon is attached to different $P$-wave $Q \bar{Q}$ pairs, a feature that is independent of the initial state and does not require the two quark pairs to have the same flavor, we conclude that there will be similar uncanceled IR singularities in NRQCD calculations of $J / \psi$ pair and $J / \psi+\Upsilon$ associated hadroproduction at NLO in $\alpha_{s}$. However, the structure of the uncanceled IR singularities will generally be more complicated there because more channels are involved. For instance, in the case of $g g \rightarrow c \bar{c}\left({ }^{3} P_{J_{c}}^{[8]}\right)+b \bar{b}\left({ }^{3} P_{J_{b}}^{[8]}\right)+g$, there will be four possible interferences of the four pairings $c \bar{c}\left({ }^{3} S_{1}^{[1]}\right)+b \bar{b}\left({ }^{3} P_{J_{b}}^{[8]}\right)$, $c \bar{c}\left({ }^{3} S_{1}^{[8]}\right)+b \bar{b}\left({ }^{3} P_{J_{b}}^{[8]}\right), c \bar{c}\left({ }^{3} P_{J_{c}}^{[8]}\right)+b \bar{b}\left({ }^{3} S_{1}^{[1]}\right)$, and $c \bar{c}\left({ }^{3} P_{J_{c}}^{[8]}\right)+$ $b \bar{b}\left({ }^{3} S_{1}^{[8]}\right)$, which yield uncanceled IR singularities.

The above two examples clearly demonstrate that NRQCD factorization as we know it is spoiled by uncanceled IR singularities for inclusive production and decay processes involving two (ore more) heavy quarkonia. At this point, we recall that factorization implies a complete separation of perturbative and nonperturbative effects. In the context of the NRQCD factorization framework, one is thus led to find a concept how to separate the problematic IR-singular terms, like $\Gamma_{3}^{\text {div }}\left(J_{c}\right)$ and $\sigma_{3}^{\text {div }}\left(J_{2}\right)$, into contributions pertaining to the hard- and soft-scale regimes. The creation and annihilation of heavy-quark pairs clearly take place at short distances. To describe such processes involving two heavy-quark pairs, it is natural to consider products of four heavy-quark fields. Since the two heavyquark pairs cannot be at rest simultaneously, we adopt the covariant form of the NRQCD Lagrangian, which at LO reads [26]:

$$
\begin{aligned}
\mathcal{L}_{\mathrm{NRQCD}}^{\mathrm{LO}}= & \bar{\psi}_{v}\left(i v \cdot D+\frac{\left(i D_{\top}^{\mu}\right)\left(i D_{\top \mu}\right)}{2 m}\right) \psi_{v} \\
& +\bar{\chi}_{v}\left(-i v \cdot D+\frac{\left(i D_{\top}^{\mu}\right)\left(i D_{\top \mu}\right)}{2 m}\right) \chi_{v},
\end{aligned}
$$

where $m$ is the heavy-quark mass, $v^{\mu}=P^{\mu} /(2 m)$ with $P^{\mu}$ being the four-momentum of the $Q \bar{Q}$ pair, $\psi_{v}$ and $\chi_{v}$ are the
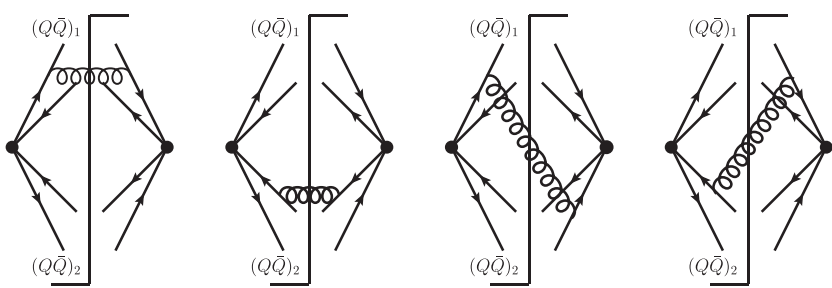

FIG. 3. Sample diagrams for one-loop corrections to the annihilation or creation of two heavy-quark pairs $Q_{1} \bar{Q}_{1}$ and $Q_{2} \bar{Q}_{2}$. The solid dots represent four-quark vertices.

nonrelativistic four-component heavy-quark and -antiquark fields, satisfying $\not \psi_{v}=\psi_{v}$ and $\not \chi_{v}=-\chi_{v}, D^{\mu}$ is the covariant derivative, and $a_{\top}^{\mu}=a^{\mu}-v^{\mu} v \cdot a$ for any fourvector $a^{\mu}$. The relative momentum $q^{\mu}$ between $Q$ and $\bar{Q}$ corresponds to $i \partial_{\top}^{\mu} \psi_{v}$.

Representative diagrams for one-loop corrections to the annihilation or creation of two heavy-quark pairs, $Q_{1} \bar{Q}_{1}$ and $Q_{2} \bar{Q}_{2}$, are depicted in Fig. 3. In the first two panels, the soft gluon interconnects the same $Q \bar{Q}$ pair. This corresponds to the product of two four-quark operators, which receive QCD corrections separately. The missing link needed to remove the leftover IR singularities is depicted in the last two panels. Here, the two $Q \bar{Q}$ pairs cross talk by exchanging a soft gluon and so form a joint structure, namely an eight-quark operator.

In the following, we refer to $b \bar{b}\left({ }^{3} P_{J_{b}}^{[8]}\right) \rightarrow c \bar{c}\left({ }^{3} P_{J_{c}}^{[1]}\right)+g g$ as process $\mathrm{A}$ and to $e^{+} e^{-} \rightarrow c \bar{c}\left({ }^{3} P_{J_{1}}^{[8]}\right)+c \bar{c}\left({ }^{3} P_{J_{2}}^{[1]}\right)+g$ as process $\mathrm{B}$ and generically denote the total and relative four-momenta of $Q_{i} \bar{Q}_{i}$ as $P_{i}$ and $q_{i}$, respectively. To generate the appropriate interference parts at one loop for two $P$-wave $Q \bar{Q}$ states, we need the new operators $\psi_{b, v_{1}} \mathcal{K}^{\mu_{1} \nu_{1}} T^{a_{1}} \bar{\chi}_{b, v_{1}} \bar{\psi}_{c, v_{2}} \gamma_{T}^{\nu_{2}} T^{a_{2}} \chi_{c, v_{2}}, \quad \psi_{b, v_{1}} \gamma_{T}^{\nu_{1}} T^{a} \bar{\chi}_{b, v_{1}} \bar{\psi}_{c, v_{2}} \times$ $\mathcal{K}_{J_{c}}^{\mu_{2} \nu_{2}} \chi_{c, v_{2}}$ and their charge conjugates for process $\mathrm{A}$ and $\bar{\psi}_{c, v_{1}} \mathcal{K}^{\mu_{1} \nu_{1}} T^{a_{1}} \chi_{c, v_{1}} \bar{\psi}_{c, v_{2}} \gamma_{T}^{\nu_{2}} T^{a_{2}} \chi_{c, v_{2}}, \bar{\psi}_{c, v_{1}} \gamma_{T}^{\nu_{1}} \chi_{c, v_{1}} \bar{\psi}_{c, v_{2}} \times$ $\mathcal{K}_{J_{2}}^{\mu_{2} \nu_{2}} \chi_{c, v_{2}}$ and their charge conjugates for process B, where $\mathcal{K}_{0}^{\mu \nu}=\left(g^{\mu \nu}-v^{\mu} v^{\nu} / \sqrt{3}\right)\left[-(i / 2) \stackrel{\leftrightarrow}{\boldsymbol{D}}_{\top}\right], \mathcal{K}_{1}^{\mu \nu}=(-i / 2)\left(\stackrel{\leftrightarrow}{\boldsymbol{D}}_{\top}^{[\mu} \gamma_{\top}^{\nu]}\right)$, $\mathcal{K}_{2}^{\mu \nu}=(-i / 2)\left(\stackrel{\leftrightarrow}{D}_{\top}^{(\mu} \gamma_{\top}^{\nu)}\right)$, and $\mathcal{K}^{\mu \nu}=(-i / 2)\left(\stackrel{\leftrightarrow}{D}_{\top}^{\mu} \gamma_{T}^{\nu}\right)$ with $a^{[\mu} b^{\nu]}=\frac{1}{2}\left(a^{\mu} b^{\nu}-a^{\nu} b^{\mu}\right)$ and $a^{(\mu} b^{\nu)}=\frac{1}{2}\left(a^{\mu} b^{\nu}+a^{\nu} b^{\mu}\right)-$ $\left(g^{\mu \nu}-v^{\mu} v^{\nu} / 3\right) a \cdot b$.

Using the Feynman rules derived from Eq. (6) in connection with the new operators introduced above, we are now in a position to evaluate the last two Feynman diagrams in Fig. 3. Although $m_{c}$ is about three times smaller than $m_{b}$, we assume that $m_{c} \gg m_{b} v_{b}$ to ensure that the soft region of bottomonium is sufficiently separated from the hard region of charmonium so that the nonrelativistic approximation still applies to the latter. Working in covariant gauge, we show that the results are gauge independent. The details of our calculation will be presented elsewhere [27]. For space limitation, here we merely explain how to perform the loop integrations, 
taking the third diagram in Fig. 3 as an example and working in Feynman gauge. The arising loop integral reads

$$
I=-i g_{s}^{2} \mu^{4-D} \int \frac{d^{D} l}{(2 \pi)^{D}} \times \frac{\left[v_{1}+\frac{\left(2 q_{1}+l\right)_{T}}{2 m_{1}}\right]\left[v_{2}+\frac{\left(2 q_{2}+l\right)_{T}}{2 m_{2}}\right]}{l^{2}\left[l \cdot v_{1}+\frac{\left(l+q_{1}\right)_{T}^{2}}{2 m_{1}}\right]\left[l \cdot v_{2}+\frac{\left(l+q_{2}\right)_{T}^{2}}{2 m_{2}}\right]} .
$$

Expanding the heavy-quark propagators in $1 / m_{i}$ and dropping terms of order $1 / m_{i}^{2}$ and higher, which contribute at higher orders in $v_{i}^{2}$, we obtain

$$
\begin{aligned}
I= & -\frac{i g_{s}^{2} \mu^{4-D}}{m_{1} m_{2}} \int \frac{d^{D} l}{(2 \pi)^{D}} \frac{1}{l^{2}}\left[\frac{q_{1} \cdot q_{2}}{\left(l \cdot v_{1}\right)\left(l \cdot v_{2}\right)}-\frac{\left(l \cdot q_{1}\right)\left(v_{1} \cdot q_{2}\right)}{\left(l \cdot v_{1}\right)^{2}\left(l \cdot v_{2}\right)}-\frac{\left(l \cdot q_{2}\right)\left(v_{2} \cdot q_{1}\right)}{\left(l \cdot v_{1}\right)\left(l \cdot v_{2}\right)^{2}}+\frac{\left(v_{1} \cdot v_{2}\right)\left(l \cdot q_{1}\right)\left(l \cdot q_{2}\right)}{\left(l \cdot v_{1}\right)^{2}\left(l \cdot v_{2}\right)^{2}}\right]+I_{0} \\
= & \frac{\alpha_{s} \mu^{4-D}}{\pi m_{1} m_{2}}\left(\frac{1}{\epsilon_{\mathrm{UV}}}-\frac{1}{\epsilon_{\mathrm{IR}}}\right)\left[q_{1} \cdot q_{2} \times \frac{\ln \left(\omega+\sqrt{\omega^{2}-1}\right)-\omega \sqrt{\omega^{2}-1}}{2\left(\omega^{2}-1\right)^{3 / 2}}+\left(v_{1} \cdot q_{2}\right)\left(v_{2} \cdot q_{1}\right)\right. \\
& \left.\times \frac{\left(\omega^{2}+2\right) \sqrt{\omega^{2}-1}-3 \omega \ln \left(\sqrt{\omega^{2}-1}+\omega\right)}{2\left(\omega^{2}-1\right)^{5 / 2}}\right]+I_{0},
\end{aligned}
$$

where $\omega=v_{1} \cdot v_{2}$ and $I_{0}$ includes irrelevant terms that will cancel in the sum over all diagrams. The ultraviolet singularities are removed via operator renormalization. Multiplying Eq. (8) with the corresponding SDCs, which is the interference of $b \bar{b}\left({ }^{3} P_{J_{b}}^{[8]}\right) \rightarrow c \bar{c}\left({ }^{3} S_{1}^{[8]}\right)+g$ and $b \bar{b}\left({ }^{3} S_{1}^{[8]}\right) \rightarrow c \bar{c}\left({ }^{3} P_{J_{c}}^{[1]}\right)+g$ for process $\mathrm{A}$ and the interference of $e^{+} e^{-} \rightarrow c \bar{c}\left({ }^{3} P_{J_{1}}^{[8]}\right)+c \bar{c}\left({ }^{3} S_{1}^{[8]}\right)$ and $e^{+} e^{-} \rightarrow$ $c \bar{c}\left({ }^{3} S_{1}^{[1]}\right)+c \bar{c}\left({ }^{3} P_{J_{2}}^{[1]}\right)$ for process $\mathrm{B}$, and decomposing the tensor and color structure into the basis of the total-angularmomentum and color states, we find that the IR-singular parts exactly match those in $\Gamma_{3}^{\operatorname{div}}\left(J_{c}\right)$ and $\sigma_{3}^{\text {div }}\left(J_{2}\right)$. We wish to emphasize that the loop integrals are process independent, although they depend on $v_{1} \cdot v_{2}$.

In summary, we discovered a surprising loophole in the standard formulation of the NRQCD factorization approach that manifests itself in the failure of IR cancelation in the presence of two (or more) $P$-wave $Q \bar{Q}$ Fock states. This inevitably causes NRQCD factorization to break down for any decay or production process involving two (or more) heavy quarkonia at a certain order of $v_{Q}^{2}$. We illustrated this for two phenomenologically important example processes, $\Upsilon \rightarrow \chi_{c J}+X$ and $e^{+} e^{-} \rightarrow J / \psi+\chi_{c J}+X$, at NLO in $\alpha_{s}$. As a solution to this problem, we introduced new types of operators and demonstrated that their NLO corrections precisely reproduced the uncanceled IR singularities, which may thus be attributed to the soft regime of NRQCD. This implies that it is possible to generalize the factorization formalism within the very same NRQCD effective field theory so as to allow for the successful theoretical description of processes involving two (or more) heavy quarkonia. The explicit construction of such a generalized NRQCD factorization formalism and its applications to heavyquarkonium phenomenology are left for future work.
We thank G. T. Bodwin and E. Braaten for very useful comments. This work was supported in part by BMBF Grant No. 05H15GUCC1. The work of X.P.W. was supported in part by Chinese Scholarship Council Scholarship No. 201404910576.

[1] R. Barbieri, R. Gatto, and E. Remiddi, Phys. Lett. 61B, 465 (1976); Nucl. Phys. B162, 220 (1980); R. Barbieri, M. Caffo, R. Gatto, and E. Remiddi, ibid. B192, 61 (1981).

[2] G. Bélanger and P. Moxhay, Phys. Lett. B 199, 575 (1987); L. Bergström and P. Ernström, ibid. 267, 111 (1991).

[3] G. T. Bodwin, E. Braaten, and G. P. Lepage, Phys. Rev. D 51, 1125 (1995); 55, 5853(E) (1997).

[4] W. E. Caswell and G. P. Lepage, Phys. Lett. 167B, 437 (1986).

[5] G. P. Lepage, L. Magnea, C. Nakhleh, U. Magnea, and K. Hornbostel, Phys. Rev. D 46, 4052 (1992).

[6] H.-W. Huang and K.-T. Chao, Phys. Rev. D 54, 3065 (1996); 56, 7472(E) (1997); 60, 079901(E) (1999); 54, 6850 (1996); 56, 1821(E) (1997); A. Petrelli, Phys. Lett. B 380, 159 (1996); A. Petrelli, M. Cacciari, M. Greco, F. Maltoni, and M. L. Mangano, Nucl. Phys. B514, 245 (1998); M. Klasen, B. A. Kniehl, L. N. Mihaila, and M. Steinhauser, ibid. B713, 487 (2005); Phys. Rev. D 71, 014016 (2005).

[7] Y. Fan, Z.-G. He, Y.-Q. Ma, and K.-T. Chao, Phys. Rev. D 80, 014001 (2009); Z.-G. He, Y. Fan, and K.-T. Chao, ibid. 81, 074032 (2010); Y. Fan, J.-Z. Li, C. Meng, and K.-T. Chao, ibid. 85, 034032 (2012); S.-J. Sang, J.-Z. Li, C. Meng, and K.-T. Chao, ibid. 91, 114023 (2015).

[8] Z.-Z. Song, C. Meng, Y.-J. Gao, and K.-T. Chao, Phys. Rev. D 69, 054009 (2004).

[9] G. T. Bodwin, X. Garcia i Tormo, and J. Lee, Phys. Rev. Lett. 101, 102002 (2008); Phys. Rev. D 81, 114014 (2010).

[10] R. Fulton et al. (CLEO Collaboration), Phys. Lett. B 224, 445 (1989). 
[11] H. Albrecht et al. (ARGUS Collaboration), Z. Phys. C 55, 25 (1992).

[12] R. A. Briere et al. (CLEO Collaboration), Phys. Rev. D 70, 072001 (2004).

[13] C. P. Shen et al. (Belle Collaboration), Phys. Rev. D 93, 112013 (2016).

[14] S. Jia et al. (Belle Collaboration), Phys. Rev. D 95, 012001 (2017).

[15] V. M. Abazov et al. (D0 Collaboration), Phys. Rev. D 90, 111101(R) (2014); Phys. Rev. Lett. 116, 082002 (2016).

[16] R. Aaij et al. (LHCb Collaboration), Phys. Lett. B 707, 52 (2012); J. High Energy Phys. 06 (2017) 047.

[17] V. Khachatryan et al. (CMS Collaboration), J. High Energy Phys. 09 (2014) 094; 05 (2017) 013.

[18] M. Aaboud et al. (ATLAS Collaboration), Eur. Phys. J. C 77, 76 (2017).

[19] Z.-G. He and B. A. Kniehl, Phys. Rev. Lett. 115, 022002 (2015).
[20] C. H. Kom, A. Kulesza, and W. J. Stirling, Phys. Rev. Lett. 107, 082002 (2011); S. P. Baranov, A. M. Snigirev, N.P. Zotov, A. Szczurek, and W. Schäfer, Phys. Rev. D 87, 034035 (2013).

[21] H. D. Trottier, Phys. Lett. B 320, 145 (1994); M. Napsuciale, Phys. Rev. D 57, 5711 (1998); Z.-G. He and J.-X. Wang, ibid. 81, 054030 (2010); 82, 094033 (2010).

[22] K. Cheung, W.-Y. Keung, and T. C. Yuan, Phys. Rev. D 54, 929 (1996).

[23] L.-P. Sun, H. Han, and K.-T. Chao, Phys. Rev. D 94, 074033 (2016); H.-S. Shao and Y.-J. Zhang, Phys. Rev. Lett. 117, 062001 (2016).

[24] Y.-J. Li, G.-Z. Xu, K.-Y. Liu, and Y.-J. Zhang, J. High Energy Phys. 07 (2013) 051.

[25] Y. Feng, Z. Sun, and H.-F. Zhang, Eur. Phys. J. C 77, 221 (2017).

[26] A. V. Manohar, Phys. Rev. D 56, 230 (1997).

[27] Z.-G. He, B. A. Kniehl, and X.-P. Wang (to be published). 